\title{
Facile one-step solid-state reaction to synthesis of hafnium carbide nanoparticles at low temperature
}

\author{
Liangbiao WANG, Wanjun XI, Tao MEI*, Yibing CAI*, ${ }^{*}$, Juanjuan LU, Dejian ZHAO, \\ Hongying HUANG, Weiqiao LIU and Quanfa ZHOU* \\ School of Chemistry and Environment Engineering, Jiangsu Engineering Center of E-waste Resource Recycling, \\ Jiangsu University of Technology, Changzhou 213001, P. R. China \\ ${ }^{*}$ Hubei Collaborative Innovation Center for Advanced Organic Chemical Materials, Ministry of Education, Key Laboratory for \\ the Green Preparation and Application of Functional Materials, School of Materials Science and Engineering, Hubei University, \\ Wuhan 430062, P. R. China \\ ** Key Laboratory of Eco-textiles, Ministry of Education, Jiangnan University, Wuxi 214122, P. R. China
}

\begin{abstract}
Usually, the synthetic process of hafnium carbide (HfC) by using hafnium dioxide as hafnium source requires high reaction temperature. In this study, we have successfully synthesized HfC nanoparticles by a solid state reaction of hafnium dioxide, lithium carbonate and metallic magnesium in a stainless-steel autoclave at $700^{\circ} \mathrm{C}$ for $10 \mathrm{~h}$. The X-ray powder diffraction pattern reveals that the obtained sample is cubic phase $\mathrm{HfC}$ with lattice constant of $a=4.633 \AA$. Scanning electron microscope images show that the obtained HfC sample is composed of uniform particles with an average size of about $10 \mathrm{~nm}$. Furthermore, the formation mechanism and oxidation resistance of the HfC nanoparticles have been investigated.
\end{abstract}

(C2017 The Ceramic Society of Japan. All rights reserved.

Key-words : Carbide, Solid state reaction, Nanoparticles, XRD

[Received July 3, 2017; Accepted August 7, 2017]

\section{Introduction}

In the past decades, there has been great interest in the synthesis and characterization of transition metal carbides due to their excellent properties, such as high melting point, excellent chemical stability, high hardness, low electric resistivity, and high wear resistance. ${ }^{1), 2)}$ The transition metal carbides have a wide range of applications in many fields for their excellent physical properties. ${ }^{3)}$ For example, titanium carbide can be using as advanced ceramics for wear resistance and aerospace applications. ${ }^{4)}$ Molybdenum carbide can be used as a hydrogen evolution catalyst. ${ }^{5)}$ Besides, $\mathrm{NbC}$ and $\mathrm{TaC}$ are superconductors.. ,7)

Hafnium carbide, one of the transition metal carbides, has a melting point of $3887^{\circ} \mathrm{C}$, low electric resistivity, and high wear resistance. ${ }^{8)}$ Very recently, hafnium carbide is thought to be able to uptake and activate $\mathrm{CO}_{2}$ on their most-stable (001) surfaces with considerable adsorption strength by means of density functional theory calculations. ${ }^{9)}$ However, the synthesis of the hafnium carbide at low temperature is especially difficult. Therefore, more and more efforts are made to develop novel synthetic routes of the hafnium carbide. Up to now, a number of research groups have reported the preparation of nanostructure hafnium carbide with varied morphologies (for instance, nanoparticles, nanowires, nanobelts). HfC nanopowders have been synthesized by the carbothermal reduction reaction of hafnium dioxide and carbon at $1475^{\circ} \mathrm{C} .{ }^{10)}$ Chang and co-workers have synthesized the cubic early transition metal carbides from metal chlorides and nbutyllithium at $800-1000^{\circ} \mathrm{C} .{ }^{11)}$ Solid-state metathesis route of $\mathrm{CaC}_{2}$ and metal halides can be used to synthesize transition metal carbide. ${ }^{12), 13)} \mathrm{HfC}$ powders were synthesized by carthermal

\footnotetext{
Corresponding author: Y. Cai; E-mail: yibingcai@jiangnan.edu.cn

* Corresponding author: Q. Zhou; E-mail: labzqf@just.edu.cn
}

reduction reaction using fine-scale carbon/metal oxide mixtures as the starting materials at $1500^{\circ} \mathrm{C} .{ }^{14)} \mathrm{HfC}$ can also be obtained by reaction of carbon and metallic hafnium or hafnium hydride. ${ }^{15), 16)}$ Abdelkader and co-workers have synthesized HfC nanopowders by the electro-deoxidation of $\mathrm{HfO}_{2}$-carbon precursors in molten chloride. ${ }^{17)}$ One-dimensional HfC nanostructure (nanobelts, nanowires, nanotubes, microcrystal chains) have been prepared by chemical vapor deposition method. ${ }^{18)-21)}$

The carbothermic reduction of hafnium dioxide has been applied to produce $\mathrm{HfC}$ because $\mathrm{HfO}_{2}$ as hafnium source is the cheap raw materials. However, the obtained HfC product (synthesized by carbothermic reduction of $\mathrm{HfO}_{2}$ ) has relatively large particle sizes and the process require high temperatures and long reaction times. Therefore, to seek a new synthetic method to prepare $\mathrm{HfC}$ nanomaterials by using $\mathrm{HfO}_{2}$ as hafnium source at a low temperature is a challenging subject. Herein, we report a novel solid-state route to the preparation of $\mathrm{HfC}$ nanoparticles by using hafnium dioxide, lithium carbonate and metallic magnesium as starting materials in a stainless-steel autoclave at $700^{\circ} \mathrm{C}$.

\section{Experimental}

All the chemical reagents used in our experimental were analytical purity, and were purchased from Shanghai Chemical Reagents Company. In a typical procedure, metallic magnesium $(0.60 \mathrm{~g})$, lithium carbonate $(0.20 \mathrm{~g})$ and hafnium dioxide $(0.42 \mathrm{~g})$ were mixed in an agate mortar and added into a stainless-steel autoclave of $20 \mathrm{~mL}$ capacity. The autoclave was sealed and put into an electric furnace. The temperature of the electric furnace was raised from room temperature to $700^{\circ} \mathrm{C}$ with a rate of $10^{\circ} \mathrm{C} / \mathrm{min}$ and maintained at $700^{\circ} \mathrm{C}$ for $10 \mathrm{~h}$. After the autoclave was cooled to room temperature naturally, the dark precipitate in the autoclave was collected and washed with hydrochloric acid $(1.0 \mathrm{~mol} / \mathrm{L})$, distilled water and ethanol for several times to 
remove the impurities. The final sample was then dried under vacuum at $50^{\circ} \mathrm{C}$ for $10 \mathrm{~h}$ for further characterization.

The X-ray powder diffraction (XRD) analysis was recorded with a Philips X'pert X-ray diffractometer equipped with $\mathrm{Cu} \mathrm{K} \alpha$ radiation $(\lambda=1.54178 \AA)$. The scanning electron microscope (SEM) images were taken on JEOL-JSM-6700F field emission electron microscope. Transmission electron microscopy (TEM) and high-resolution transmission electron microscopy (HR-TEM) images were taken on a Hitachi Model H-800 transmission electron microscope and JEOL-2010 high-resolution transmission electron microscope, respectively. Thermogravimetric analysis (TGA) profile was collected with a Shimadzu-50 thermoanalyzer apparatus under flowing air.

\section{Results and discussion}

$\mathrm{X}$-ray power diffraction analysis is used to investigate the phase of the obtained materials. XRD pattern of the obtained sample is shown in Fig. 1. All the five reflection peaks in XRD pattern can be indexed to the cubic phase HfC, with lattice constant of $a=4.633 \AA$, which agrees with the reported data (JCPDS card, No. 65-0975, $a=4.637 \AA$ ). The size of the obtained HfC has been estimated to be $10 \mathrm{~nm}$ according to the calculation result of the Scherrer formula.

The morphology of the obtained HfC sample is further analyzed by SEM and TEM. SEM images of the obtained sample are shown in Figs. 2(a) and 2(b). It can be seen that the obtained HfC sample is composed of uniform particles with an average size of about $10 \mathrm{~nm}$, which is consistent with calculation result of the Scherrer formula. The TEM image further indicates that the obtained sample is composed of uniform nanoparticles as shown in Fig. 2(c). The HR-TEM image of the obtained HfC sample is shown in Fig. 2(d). The interplanar spacing is about 0.27 $\mathrm{nm}$, which corresponds to (111) spacing of cubic phase hafnium carbide.

A series of experiments are carried out by altering experimental parameters of the process to study the formation of the cubic phase hafnium carbide. It is found that the reaction temperature and reaction time have great influence on the formation of the hafnium carbide. No hafnium carbide could be obtained by the chemical reaction of hafnium dioxide, lithium carbonate and metallic magnesium at the temperature of $600^{\circ} \mathrm{C}$. When the temperature is $650^{\circ} \mathrm{C}$, HfC with low crystallinity is detected in the XRD pattern of the product. When the reaction temperature is above $750^{\circ} \mathrm{C}$ resulted in the size of the obtained HfC particles increasing. An optimum reaction temperature for the preparation

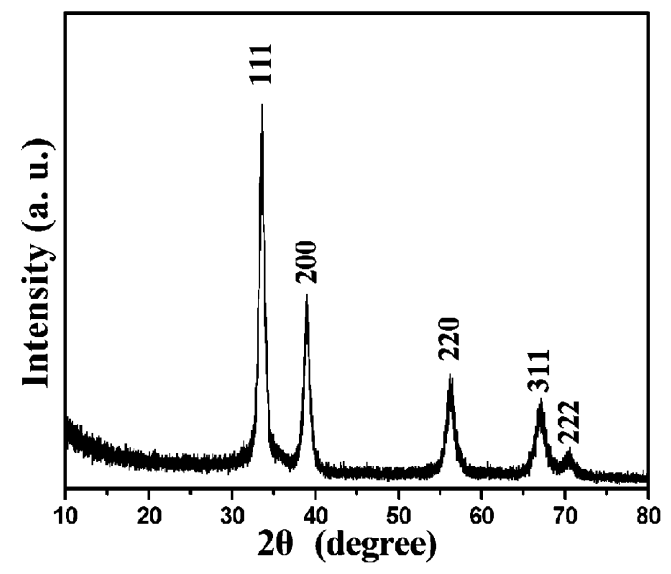

Fig. 1. XRD pattern of the obtained sample. is about $700^{\circ} \mathrm{C}$. If the reaction time is shorten than $5 \mathrm{~h}$, the reaction becomes incomplete at $700^{\circ} \mathrm{C}$. The reaction time in the range of $6-10 \mathrm{~h}$ at $700^{\circ} \mathrm{C}$ does not effect the size of the product. When metallic lithium is used instead of metallic magnesium, HfC nanoparticles can also be obtained by the chemical reaction of hafnium dioxide, lithium carbonate and metallic lithium at $700^{\circ} \mathrm{C}$ through similar process. When lithium carbonate and metallic magnesium (excessive) are added into the autoclave and kept at $700^{\circ} \mathrm{C}$ for $10 \mathrm{~h}$, we find that carbon can be obtained by the reaction of metallic magnesium and lithium carbonate. The reaction between metallic magnesium and lithium carbonate can be describe as follows:

$$
\mathrm{Li}_{2} \mathrm{CO}_{3}+3 \mathrm{Mg}=3 \mathrm{MgO}+2 \mathrm{Li}+\mathrm{C}
$$

According to free energy calculations, the Eq. (1) is highly exothermic $\left(\Delta_{\mathrm{r}} H_{\mathrm{m}}=-587.85 \mathrm{~kJ} / \mathrm{mol}\right)$. When hafnium dioxide and metallic magnesium (excessive) are added into the autoclave and kept at $700^{\circ} \mathrm{C}$ for $10 \mathrm{~h}$, we find that metallic magnesium don't react with hafnium dioxide at $700^{\circ} \mathrm{C}$. Therefore, we think that a great deal of heat [generated from the Eq. (1)] promote hafnium dioxide to take part in the chemical reaction and form HfC nanoparticles. The reaction used in our approach can be expressed as follows:

$$
\mathrm{HfO}_{2}+\mathrm{Li}_{2} \mathrm{CO}_{3}+5 \mathrm{Mg}=5 \mathrm{MgO}+2 \mathrm{Li}+\mathrm{HfC}
$$

The oxidation resistance of the obtained HfC sample is investigated under flowing air from 25 to $1000^{\circ} \mathrm{C}$ with a heating ramp rate of $10^{\circ} \mathrm{C} / \mathrm{min}$. The TGA curve of the obtained HfC sample is shown in Fig. 3. The TGA curve reveals that the weight gain of the sample has not changed significantly below $250^{\circ} \mathrm{C}$, which indicates the obtained $\mathrm{HfC}$ sample is stable below $250^{\circ} \mathrm{C}$ in air atmosphere. When the temperature is between 250 and $620^{\circ} \mathrm{C}$, the weight of the sample has increased, which proves that the sample have been oxidized by oxygen with simultaneous ejection of carbon ( $\mathrm{HfC}+\mathrm{O}_{2}=\mathrm{HfO}_{2}+\mathrm{C}$ ). However, the weight gain of the sample becomes smaller and smaller when the temperature is between 620 and $680^{\circ} \mathrm{C}$, which induced by the oxidation of carbon in the process $\left(\mathrm{C}+\mathrm{O}_{2}=\mathrm{CO}_{2}\right)$. A similar phenomenon is found in our previous work. ${ }^{22}$ When the temperature exceeds $680^{\circ} \mathrm{C}$, the weight gain remains almost constant. These results illustrate that the obtained HfC nanoparticles in our method have good thermal stability below $250^{\circ} \mathrm{C}$ under air flowing.
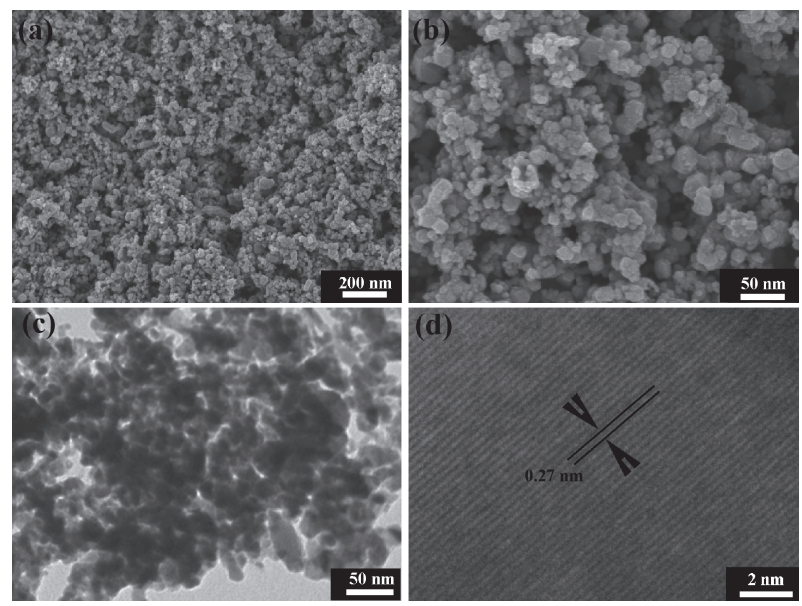

Fig. 2. (a) A typical low-magnification SEM image; (b) A typical highmagnification SEM image; (c) TEM image; (d) HR-TEM image of the obtained sample. 


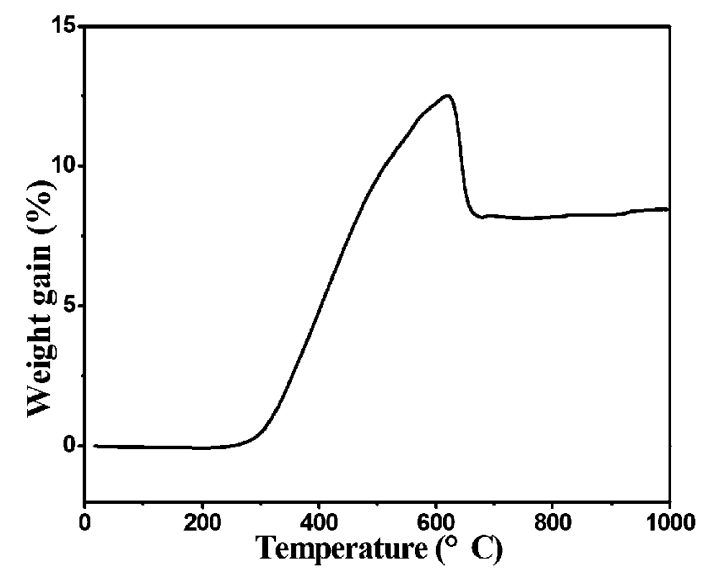

Fig. 3. TGA curve heated in flowing air for the obtained HfC sample.

\section{Conclusions}

In summary, we describe a novel solid-state route to synthesize HfC nanoparticles under mild reaction conditions. HfC nanoparticles have been synthesized by using hafnium dioxide, lithium carbonate and metallic magnesium as starting materials in a stainless-steel autoclave at $700^{\circ} \mathrm{C}$. The formation mechanism and oxidation resistance of HfC have been discussed. We believe that this simple method can be used in preparation of other transition metal carbides.

Acknowledgments This work was financially supported by the Open Project Program of Key Laboratory of Eco-textiles, Ministry of Education, Jiangnan University (No. KLET1604), the National Natural Science Foundation of China (No. 21701061, 21401049), the Natural Science Foundation of Jiangsu Province (No. BK20160292), and Natural Science Foundation of the Higher Educations Institutions of Jiangsu Province (No. 16KJB150013).

\section{References}

1) H. O. Pierson, Handbook of Refractory Carbides and Nitrides, 1st Ed., Noyes Publications, New Jersey (1996).
2) A. W. Weimer, Carbide, nitride and boride materials synthesis and processing (1997).

3) E. K. Storms, The Refractory Carbides, 1st Ed., Academic Press, New York (1967).

4) R. M. Mahamood, E. T. Akinlabi, M. Shukla and S. Pityana, Mater. Design, 50, 656-666 (2013).

5) H. Wang, C. Sun, Y. J. Cao, J. T. Zhu, Y. Chen, J. Guo, J. Zhao, Y. H. Sun and G. F. Zou, Carbon, 114, 628-634 (2017).

6) S. R. Vallance, D. M. Round, C. Ritter, E. J. Cussen, S. Kingman and D. H. Gregory, Adv. Mater., 21, 4502-4504 (2009).

7) L. B. Wang, Q. W. Li, T. Mei, L. Shi, Y. C. Zhu and Y. T. Qian, Mater. Chem. Phys., 137, 1-4 (2012).

8) M. M. Opeka, I. G. Talmy, E. J. Wuchina, J. A. Zaykoski and S. J. Causey, J. Eur. Ceram. Soc., 19, 2405-2414 (1999).

9) C. Kunkel, F. Vines and F. Illas, Energy Environ. Sci., 9, 141144 (2016).

10) C. A. Wang, M. D. Sacks and Z. Yang, Ceram. Trans., 154, 27-36 (2003).

11) Y. H. Chang, C. W. Chiu, Y. C. Chen, C. C. Wu, C. P. Tsai, J. L. Wang and H. T. Chiu, J. Mater. Chem., 12, 2189-2191 (2002).

12) A. M. Nartowski, I. P. Parkin, M. MacKenzie, A. J. Craven and I. MacLeod, J. Mater. Chem., 9, 1275-1281 (1999).

13) L. B. Wang, Q. W. Li, Y. C. Zhu and Y. T. Qian, Int. J. Refract. Met. Hard Mater., 31, 288-292 (2012).

14) M. D. Sacks, C. A. Wang, Z. H. Yang and A. Jain, J. Mater. Sci., 39, 6057-6066 (2004).

15) Y. D. Blum, J. Marschall, D. Hui, B. Adair and M. Vestel, J. Am. Ceram. Soc., 91, 1481-1488 (2008).

16) M. M. Guron, M. J. Kim and L. G. Sneddon, J. Am. Ceram. Soc., 91, 1412-1415 (2008).

17) A. M. Abdelkader and D. J. Fray, J. Eur. Ceram. Soc., 32, 4481-4487 (2012).

18) S. Tian, H. J. Li, Y. L. Zhang, S. Liu, Y. X. Fu, Y. X. Li and X. F. Qiang, J. Alloy. Compd., 580, 407-411 (2013).

19) S. Tian, H. J. Li, Y. L. Zhang, J. C. Ren, X. F. Qiang and J. Wang, J. Am. Ceram. Soc., 97, 48-51 (2014).

20) S. Tian, Y. L. Zhang, J. C. Ren, X. F. Qiang, S. Y. Zhang and H. J. Li, Appl. Surf. Sci., 402, 344-351 (2017).

21) S. Tian, H. J. Li, Y. L. Zhang, S. Y. Zhang, Y. J. Wang, J. C. Ren and X. F. Qiang, J. Cryst. Growth, 384, 44-49 (2013).

22) L. B. Wang, L. L. Si, Y. C. Zhu and Y. T. Qian, Int. J. Refract. Met. Hard Mater., 38, 134-136 (2013). 